\title{
AVALIAÇÃO DA PESQUISA AGROPECUÁRIA EM COOPERATIVAS AGROINDUSTRIAIS UTILIZANDO UM MODELO DE SCORECARD DINÂMICO
}

\section{EVALUATION OF RESEARCH ON AGRICULTURE IN AGROINDUSTRIAL COOPERATIVES USING A DYNAMIC SCORECARD MODEL}

\author{
ROBERTO MAX PROTIL \\ Professor Titular da Escola de Negócios da \\ Pontifícia Universidade Católica do Paraná \\ Curitiba/PR, Brasil \\ E-mail: roberto.protil@pucpr.br
}

\author{
AMARILDO DA CRUZ FERNANDES \\ Professor Adjunto da Escola de Engenharia da \\ Universidade Federal do Rio de Janeiro \\ Rio de Janeiro/RJ, Brasil \\ E-mail: amarildo@ufrj.br
}

\author{
ALFREDO BENEDITO KUGERATSKI SOUZA \\ Mestre em Administração pela \\ Pontifícia Universidade Católica do Paraná \\ Araucaria/PR, Brasil \\ E-mail: alfredoks@gmail.com
}

Resumo

Este trabalho teve por objetivo desenvolver um instrumento de avaliação dos investimentos em pesquisa agrícola na cadeia produtiva do trigo e seu impacto na rentabilidade de cooperativas agroindustriais. Foi desenvolvido um modelo conceitual utilizando as metodologias Balanced Scorecard e Dinâmica de Sistemas, sendo que os dados e informações necessárias ao processo de modelagem foram obtidos por meio de estudo de caso e de pesquisa ação. No desenvolvimento do modelo foram inicialmente mapeados todos os processos envolvidos na pesquisa agrícola da cadeia produtiva do trigo. Posteriormente foi desenvolvido um mapa estratégico do BSC, explicitando os objetivos e indicadores da cooperativa. Finalmente usando o método da Dinâmica de Sistemas foi conduzida uma modelagem visando ampliar a visão sistêmica do problema. Esta modelagem resultou em dois diagramas: Diagrama de Enlace Causal e Diagrama de Fluxos e Estoques. 0 modelo desenvolvido neste trabalho permitiu uma melhor compreensão das complexas relações entre pesquisa e produção agrícola, facilitando assim o processo de analise e decisão de novos investimentos em pesquisa por parte dos gestores e analistas da cooperativa agroindustrial em estudo.

Palavras-chave: cooperativas agroindustriais; pesquisa agropecuária; balanced scorecard.

\begin{abstract}
The objective of this work is to develop an investment evaluation instrument for agricultural research considering the wheat productive chain and its impact in the yield of agricultural cooperatives. A conceptual model was developed using the balanced scorecard and dynamic systems methodologies. The data and necessary information to the modelling process had been gotten through action research and case study. The modelling process was initiated by the mapping of all agricultural research processes of the wheat productive chain. Later a BSC strategic map with the objectives and indicators of the cooperative in study was developed. Finally using the dynamic system approach, a modelling aiming to improve systemic view of the problem was developed. This modelling process ended up in two diagrams: Causal Loop Diagram and Flows and Supplies Diagrams. The model developed in this work improves the comprehension of the complex relations between the research and the agricultural production. Thus, it facilitates the analysis and decision process of new research investments by the studied agro-industrial cooperative analysts and managers.
\end{abstract}

Key words: agricultural cooperatives, agricultural research, balanced scorecard. 


\section{INTRODUÇÃO}

As cooperativas constituem-se como sociedades de pessoas que se unem em uma organização, visando à satisfação de necessidades comuns. Ao contrário das sociedades mercantis, nas quais o poder é proporcional ao capital, nas cooperativas o poder é igualitário, na medida em que cada cooperado exerce o direito de um único voto, independentemente do capital possuído (BIALOSKORSKI NETO, 2001). Essas organizações desempenham um papel importante para a economia brasileira, tanto referente à geração de empregos quanto pela participação nos resultados da balança comercial do país, com uma participação de 27,87\% no resultado do PIB em 2005 (ABAG, 2006).

O impacto das mudanças nos cenários econômicos e políticos afeta diretamente as organizações agroindustriais, que são o foco desta pesquisa. Como uma parte significativa da produção abastece mercados externos, qualquer alteração no cenário mundial ou na política econômica brasileira resulta em modificações do desempenho dessas organizações. Neste sentido é importante pontuar que as sociedades cooperativistas apresentam peculiaridades em sua constituição legal, com conseqüente adoção de princípios e doutrinas que exercem significativos reflexos na sua estrutura organizacional, na delegação e no exercício de poder e, conseqüentemente, na forma como ocorre o processo decisório e planejamento e execução de suas estratégias.

Assim, em termos de gestão estratégica pode-se afirmar que as cooperativas industriais enfrentam os mesmos desafios de qualquer organização. Um destes desafios é como converter a estratégia em um processo contínuo, em que a implementação da estratégia se torne uma tarefa de todos.

Foi a partir da popularização do Balanced Scorecard (BSC), desenvolvido por Kaplan e Norton em 1992, que vem se discutindo uma maneira diferente para a implementação da estratégia na organização. Essa abordagem procura incorporar uma nova visão ao problema da modelagem da estratégia, procurando explicitá-la por meio de um mapa estratégico no qual os objetivos da estratégia sejam delineados de forma muldimensional.

Muito embora o BSC tenha se constituído numa ferramenta muito útil aos gestores, já são reconhecidas algumas limitações na forma da explicitação da estratégia formal. Foi devido a essas limitações que Fernandes (2003) propôs o emprego da Dinâmica de Sistemas (DS), uma abordagem amplamente empregada na explicitação do pensamento sobre a realidade. A integração da Dinâmica de Sistemas (DS) ao Balanced Scorecard, denominada por Fernandes (2003) de Scorecard Dinâmico, visa ampliar as fronteiras do BSC para uma visão sistêmica com diferentes desdobramentos em termos de decisão e avaliação estratégica.

Portanto, devido aos problemas que as cooperativas agroindustriais estão enfrentando para avaliar os resultados alcançados com as pesquisas agropecuárias, este trabalho propõe e discute o desenvolvimento de um modelo conceitual de Balanced Scorecard integrado à Dinâmica de Sistemas, que auxilie os gestores a avaliar os resultados dos investimentos em pesquisas agropecuárias, tendo por objetivo explicitar as relações e interdependências entre as variáveis relevantes.

\section{AGRONEGÓCIO E COOPERATIVISMO}

O conceito de agronegócio representa um enfoque moderno e sistêmico, envolvendo todas as empresas que produzem, processam e distribuem produtos 
agropecuários. Esse conjunto de processos e instituições ligadas por objetivos comuns constitui um sistema que, por sua vez, engloba outros sistemas menores, ou subsistemas. 0 sistema maior é o chamado negócio agrícola, agronegócio ou agribusiness (DAVIS \& GOLBERG, 1957 apud CASTRO, 2000).

O agronegócio compõe-se de cadeias produtivas, e estas possuem, entre seus componentes, os sistemas produtivos que operam em diferentes ecossistemas ou sistemas naturais. Nesse contexto, existe um conglomerado de instituições de apoio, composto de instituições de crédito, pesquisa, assistência técnica, entre outras, e um aparato legal e normativo, exercendo forte influência no desempenho do agronegócio (CASTRO, 2000).

A percepção sobre o grande potencial econômico das cooperativas agropecuárias cresce na medida em que, em nível mundial, o agronegócio brasileiro vem conquistando importantes mercados de forma progressiva. O superávit comercial do agronegócio foi de aproximadamente US\$ 35 bilhões no acumulado de maio de 2004 a abril de 2005, número resultante das exportações de US\$ 40,6 bilhões, que está 22,4\% acima do valor alcançado no período anterior, de maio de 2003 a abril de 2004 (ABAG, 2005). Além disso, o setor de agronegócio contribuiu com $27,87 \%$ do produto interno bruto e $24 \%$ dos empregos gerados no Brasil em 2005 (ABAG, 2006).

O cooperativismo paranaense congrega mais de 348 mil pessoas, distribuídas em 209 cooperativas dos seguintes ramos: agropecuário, crédito, trabalho, educação, saúde, habitação, consumo, transporte, infra-estrutura e turismo. As receitas totais para os cooperados atingiram R\$ 18 bilhões em 2004, contra R\$15,5 bilhões no ano anterior, o que representa mais de $18 \%$ do produto interno bruto (PIB) do Estado do Paraná. Dentre esses números, tem destaque o incremento do número de cooperados, que cresceu mais de $15 \%$ em 2004 (OCEPAR, 2005).

As cooperativas agropecuárias representam cerca de $53 \%$ da economia agrícola do Estado do Paraná e participam, de forma intensa, em todo o processo de produção, beneficiamento, armazenamento, industrialização e comercialização, que conta com 74 cooperativas distribuídas por todo o território paranaense, cujos quadros sociais somavam, em 2005, 106.211 cooperados e empregavam quase 40.673 funcionários (OCEPAR, 2007).

\section{BALANCED SCORECARD}

O primeiro artigo publicado por Kaplan e Norton sobre o BSC foi "The Balanced Scorecard - Measures that Drive Performance" (Harvard Business Review, janeiro-fevereiro de 1992). Desde essa data, cresce o número de estudos e aplicações do sistema nas áreas industrial, pública e de serviços.

- Balanced Scorecard busca traduzir a visão e a estratégia das empresas num conjunto abrangente de objetivos e medidas de desempenho, servindo de base para um sistema de gestão estratégica. Todo o desenvolvimento do BSC acontecerá a partir da modelagem para traduzir a estratégia da organização num conjunto de objetivos e indicadores. Tais objetivos serão pensados a partir de 4 perspectivas: a financeira, a dos clientes, a dos processos internos e a de crescimento e aprendizado.

Dessa forma, todo objetivo ou indicador selecionado para um scorecard deve ser um elemento de uma cadeia de relações de causa e efeito que comunique o significado da estratégia da empresa. Portanto, a tradução da estratégia da organização se resumirá num conjunto de hipóteses sobre relações de causa e efeito que devem ser testadas ao longo do processo de apuração e avaliação dos resultados. Para o completo desenvolvimento do scorecard segue-se ainda a definição de metas e ações estratégicas relativas a cada 
objetivo estratégico. Assim, a etapa de modelagem da estratégia e construção do scorecard resultará num esquema que servirá como um guia do caminho a ser perseguido para atingimento das grandes diretrizes da organização.

Não obstante o sucesso do BSC há uma concordância entre os autores Richmond (1999 e 2001), Fernandes (2002), Akkermans e Oorschot (2002), LINARD et al (2001) e Todd \& Palmer (2000) de que o processo de modelagem que origina os mapas estratégicos do BSC (strategy maps) possui pouca flexibilidade para explicitar as conseqüências de eventos externos, ou mesmo os efeitos adversos de certas opções ou decisões estratégicas. Muito embora o BSC tente se apoiar no paradigma sistêmico, há um consenso, cada vez maior, de que a linguagem que ele utiliza no processo de modelagem da estratégia tem duas limitações: não consegue capturar nem a passagem do tempo, e nem a estrutura sistêmica responsável pelo comportamento da performance da estratégia. Visando superar o arcabouço linear e estático da linguagem do BSC, Fernandes (2003) propôs a incorporação da Dinâmica de Sistemas (DS) ao processo de modelagem da estratégia com o BSC.

\section{DINÂMICA DE SISTEMAS}

Faz 46 anos desde que Jay Forrester publicou o livro Industrial Dynamics, dando início à Dinâmica de Sistemas (DS). Desde então o campo se expandiu, para contemplar pesquisadores e praticantes em várias áreas do conhecimento, tais como medicina, economia, sociologia, planejamento militar, para não mencionar as várias áreas de aplicação no domínio dos negócios. Historicamente a DS integra três campos de conhecimentos: 1) a engenharia de controle e os conceitos de realimentação e autoregulação; 2) a cibernética e o papel da informação em sistemas de controle; 3) a teoria da decisão em organizações humanas.

A descoberta de que os modelos mentais influenciam a forma pela qual as pessoas enxergam a realidade tem, sem sombra de dúvidas, orientado o emprego da Dinâmica de Sistemas muito mais no intuito da explicitação do pensamento sobre a realidade, do que da obtenção de respostas numéricas para problemas bem estruturados. Nesse sentido, o mais importante no processo de uso da Dinâmica de Sistemas é a produção de insights durante o processo de modelagem, idéia fortemente identificada com a criação de conhecimento, que segundo De Geus (1994), ex-executivo da Shell, constitui-se num dos elementos centrais para a inovação e para a percepção de novas oportunidades. Segundo ele, o uso da DS no estudo de cenários contribuiu decisivamente para acelerar 3 a 4 vezes a velocidade de aprendizado e de implementação de novos sistemas internos na Shell, propiciando à empresa um preparo maior face às mudanças que ocorreram no início dos anos de 1980. Tal fato coloca a Dinâmica de Sistemas numa perspectiva diferente dos tradicionais métodos analíticos e econométricos, situando-a no coração da prática e da teoria do aprendizado organizacional, como postula Senge (1990) no livro "A Quinta Disciplina”.

\subsection{A MODELAGEM EM DINÂMICA DE SISTEMAS}

Em Dinâmica de Sistemas duas formas de modelagem são empregadas para caracterizar um sistema: a abordagem soft e hard. Um dos resultados da modelagem soft (qualitativa) da DS é a possibilidade de visualização de qualquer sistema, por meio da identificação das suas características estruturais, das relações causa-efeito-causa e das estruturas de feedback. Tal tarefa utiliza a técnica dos chamados diagramas de Enlace Causal (causal loop diagrams), como na Figura 1. 


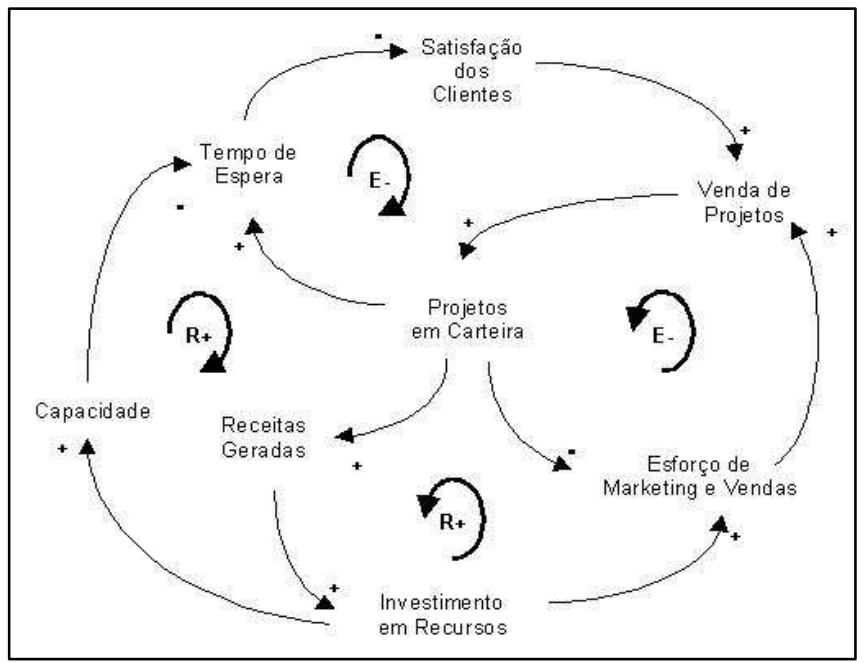

Figura 1 - DIAGRAMA DE ENLACE CAUSAL: MODELAGEM SOFT

Um segundo modo de operação, de natureza quantitativa, é a utilização das características estruturais do diagrama de Enlace Causal para desenvolver um modelo de simulação do sistema, usando a linguagem dos Fluxos e Estoques (Figura 2). Assim, os modelos de simulação podem ser usados para investigar e compreender por que certo sistema se comporta daquela maneira, permitindo, dessa forma, encontrar melhores formas de operá-lo, uma vez que se pode conhecer de antemão as conseqüências que certos eventos desencadearão no sistema. Em resumo, o emprego da modelagem da DS, consiste na explicitação dos modelos mentais coletivos acerca de uma situação, que posteriormente serão empregados para suportar qualquer processo de avaliação estratégica onde seja importante para o grupo compreender ou antever as conseqüências de certas decisões ou políticas.

FIGURA 2- DIAGRAMA DE FLUXOS E ESTOQUES: MODELAGEM HARD

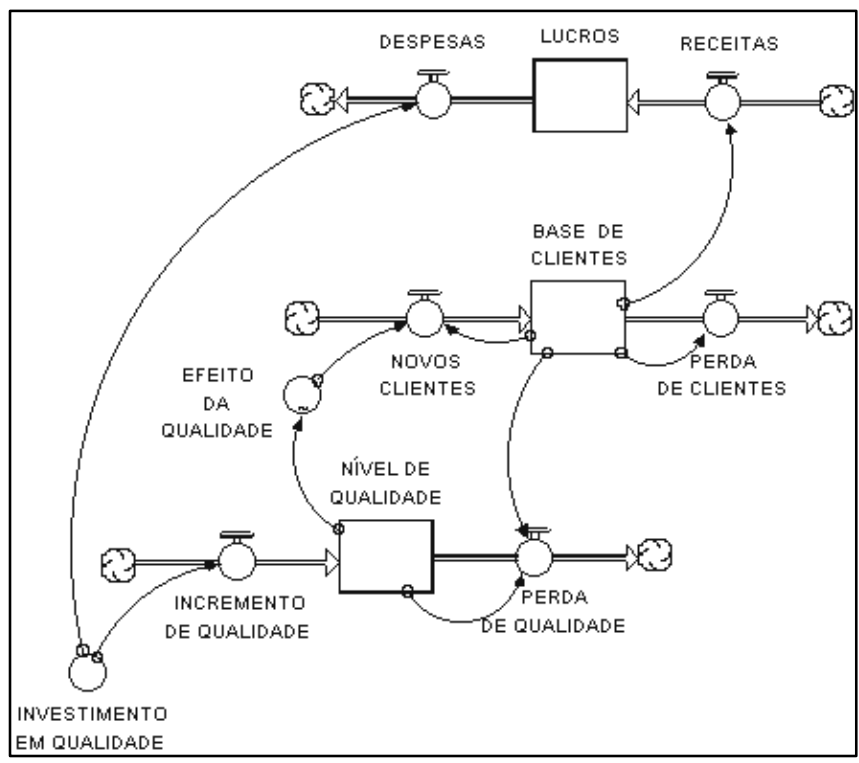




\section{METODOLOGIA}

0 problema tratado no presente trabalho refere-se aos investimentos em pesquisas agropecuárias de uma cooperativa agroindustrial e seu impacto na rentabilidade dos seus associados. Atualmente os gestores das unidades de negócio da cooperativa agroindustrial em estudo, que estão envolvidos diretamente com os investimentos em pesquisa agropecuária e a mensuração dos resultados, não possuem um sistema que demonstre os impactos desses investimentos na rentabilidade dos cooperados. Pela falta de um sistema de gestão que consiga consolidar todas as informações da unidade de pesquisa agropecuária e que apresente os resultados e impactos ao longo do tempo na estrutura da Cooperativa do caso, foi desenvolvido um modelo conceitual integrando as metodologias do BSC e da DS para explicitar as variáveis e relacionamentos importantes para o controle e monitoramento dos investimentos em pesquisa agropecuária e seu impacto na rentabilidade dos cooperados.

A metodologia adotada neste estudo foi a pesquisa-ação participante, em que todos os atores ligados à cadeia do trigo da Cooperativa Agroindustrial em estudo, situada na região centro-sul do Estado do Paraná, participaram intensamente das discussões que culminaram com a elaboração dos modelos. Foi utilizado o seguinte roteiro de pesquisa composto por quatro fases para desenvolver este estudo:

Fase exploratória: Nessa fase foram realizados três visitas a organização em questão, com o objetivo de conhecer os atores e as unidades que participariam da pesquisa. Também foram apresentados, por parte da organização, os problemas e as dificuldades de gestão da unidade responsável pelas pesquisas agropecuárias da organização. Nessas primeiras visitas foram apresentados os conceitos das duas metodologias utilizadas Balanced Scorecard (BSC) e Dinâmica de Sistemas (DS). Foram apresentados e discutidos os projetos de pesquisa em andamento, seus objetivos e possíveis retornos financeiros para a Cooperativa do caso.

Fase de pesquisa aprofundada: Com a definição do caso a ser estudado, do tema e dos objetivos da pesquisa e das Unidades de Negócios envolvidas para o desenvolvimento deste projeto, iniciaram-se os primeiros levantamentos referentes à estrutura da organização como um todo. Primeiramente, foram discutidos e verificados todos os processos internos com o objetivo de desenvolver um mapeamento desses processos da organização, e das unidades de negócios pesquisadas verificando como ocorre o processo de decisão para novos investimentos em pesquisa agropecuária, suas etapas e suas peculiaridades.

Fase de ação: Nessa fase da pesquisa, foram colocadas em prática as etapas para o desenvolvimento de instrumentos para definição dos objetivos e metas da organização necessárias para a criação do BSC que, segundo Kaplan e Norton (1997), seriam: esclarecer a estratégia para atingir a missão e visão; desenvolver os objetivos estratégicos após a tradução da estratégia; classificar todos os objetivos; verificar as inter-relações existentes; desenvolver o mapa estratégico; desdobramento dos objetivos em metas; definir os indicadores; criar um roteiro para implantação do Balanced Scorecard; com a caracterização da cadeia produtiva do trigo da Cooperativa Agroindustrial do caso foi desenvolvido o mapa estratégico na visão do Cooperado. Com a validação do mapa estratégico pelos atores envolvidos no trabalho, foi iniciada a construção do diagramas de enlace causal e estoque e fluxo para integrar a Dinâmica de Sistemas (DS) ao Balanced Scorecard (BSC).

Fase da avaliação: Essa fase teve como objetivo verificar a efetividade do trabalho em relação aos objetivos pretendidos, bem como o resgate do conhecimento que foi adquirido no processo de desenvolvimento deste trabalho. 


\section{CARACTERIZAÇÃO DA CADEIA DO TRIGO}

Neste trabalho foi estudada a cadeia produtiva do trigo na visão do cooperado da cooperativa agroindustrial em estudo. Foram levantados e estudados os principais "elos" dessa cadeia, conforme a Figura 3. Sendo observados todos os "elos" que ligam o processo de pesquisa agropecuária até o cliente/mercado. A cadeia agroalimentar pode ser definida como a seqüêencia de atividades que transformam uma commodity em produto acabado para o consumidor final, não se preocupando com a variável preço no processo de coordenação, considerando que as fronteiras podem modificar-se a longo do tempo (ROSSI \& NEVES, 2004).

Figura 3: CADEIA DO TRIGO NA VISÃO DO COOPERADO

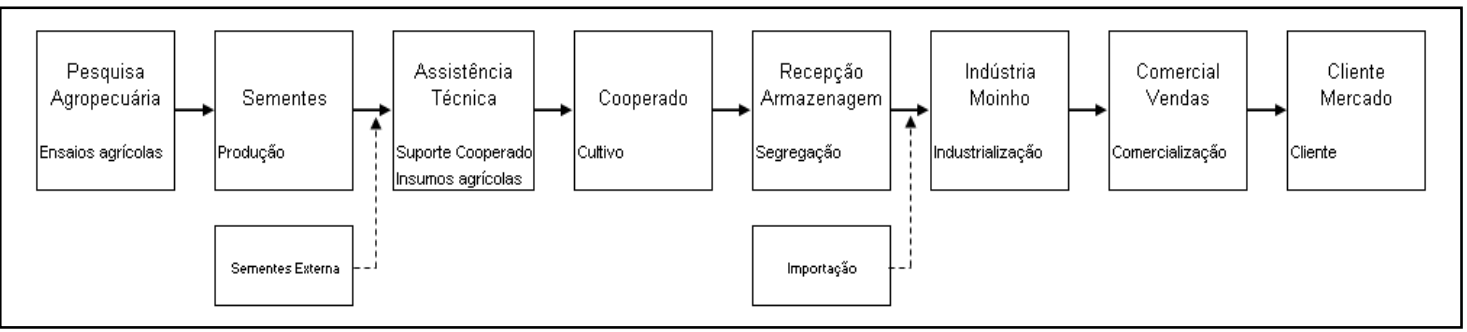

Na Figura 4 é apresentada a cadeia do trigo conforme Rossi e Neves (2004). Podese verificar, na cadeia desenvolvida, os elementos principais para uma análise descritiva, entre eles, os agentes, as relações entre eles, os setores, as organizações de suporte e o ambiente institucional (ZYLBERSTAJN, 2000).

A diferença entre a cadeia produtiva do trigo na visão do cooperado desenvolvido na Cooperativa Agroindustrial do caso em relação a cadeia apresentada por Rossi e Neves (2004) é em relação ao elo de "Pesquisas Agropecuárias" que não esta ilustrada na figura 4. 
Figura 4: A CADEIA DO TRIGO NO BRASIL

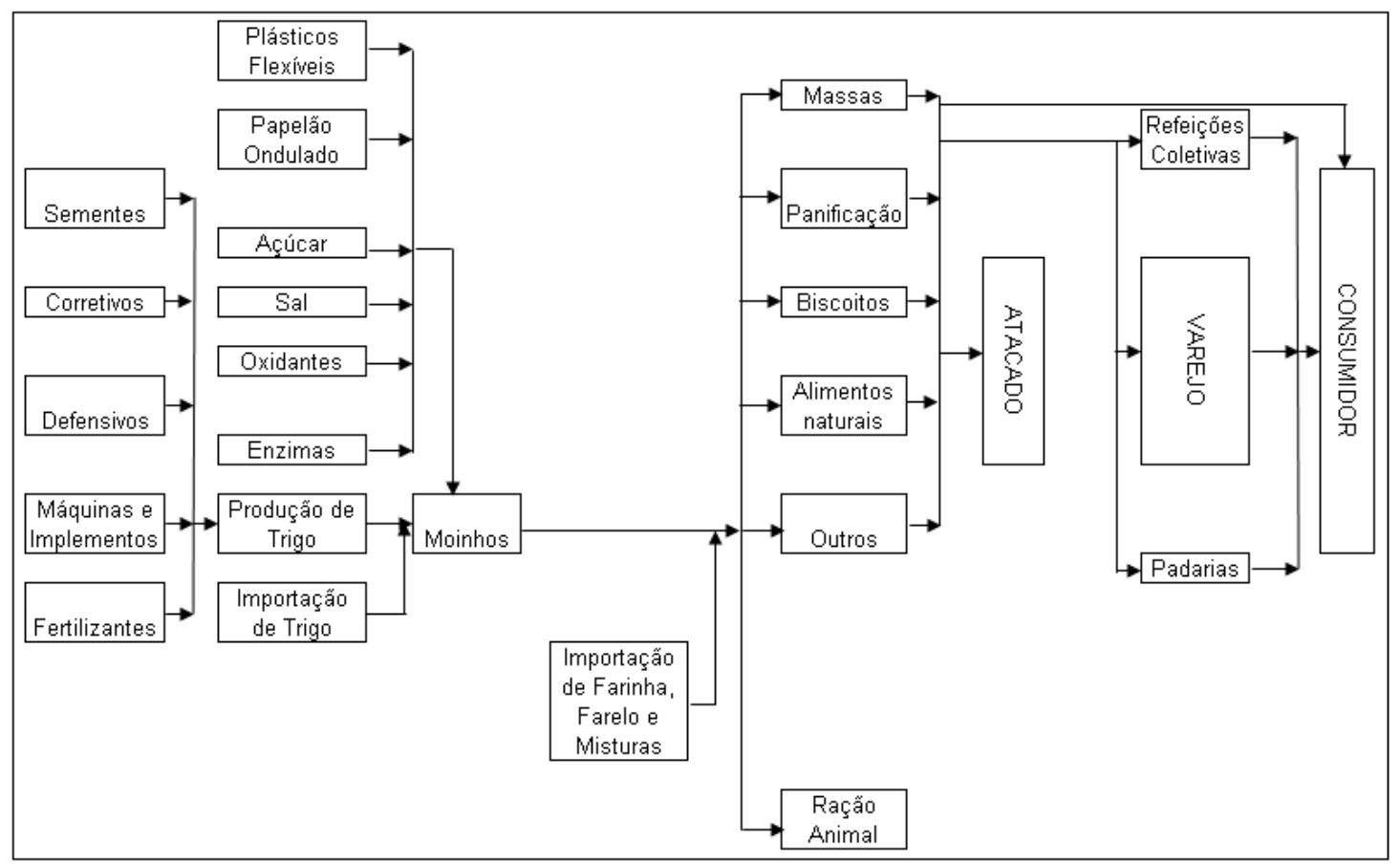

Fonte: Rossi \& Neves (2001, p. 59).

\section{ELABORAÇÃO DO MAPA ESTRATÉGICO DO BALANCED SCORECARD}

Essa etapa teve como objetivo principal a modelagem do mapa estratégico da cadeia produtiva do trigo, para explicar os objetivos estratégicos da organização e, com isso, dar suporte a um sistema de controle, acompanhamento e avaliação do desempenho da estratégia sendo desenvolvida com base a estrutura conceitual do BSC. O resultado foi o delineamento de um mapa com objetivos conectados por relações de causa e efeito, conforme a metodologia de Kaplan e Norton.

Entrevistas para a construção do BSC: Em entrevista realizada com o responsável pela unidade de pesquisa da Cooperativa e com o pesquisador da linha do trigo, com o objetivo de descrever os processos para a realização das pesquisas, as ligações existentes entre "elos" da cadeia do trigo e sua importância como unidade de negócio da cooperativa foram levantadas. Essa entrevista foi realizada em junho de 2006, com uma duração de aproximadamente três horas. Na seqüência da realização do presente trabalho foi realizada uma entrevista com o responsável da Unidade Industrialização do Trigo, Indústria-Moinho, da Cooperativa. Essa entrevista tinha como objetivo entender o processo de industrialização do trigo, o contato e relacionamento com o cliente e a identificação de processos críticos da produção industrial. Para finalizar o primeiro levantamento das informações para o desenvolvimento do mapa estratégico da cadeia do trigo, foi realizada uma entrevista com o ator do grupo de trabalho da área financeira da Cooperativa do estudo de caso. Essa entrevista teve como objetivo verificar a visão da cooperativa em relação aos investimentos em pesquisas agrícolas e a rentabilidade do cooperado e a formulações de métricas financeiras para o mapa estratégico.

Construção do modelo: Como a definição do propósito do mapa estratégico da cadeia do trigo que é o de compreender os impactos na rentabilidade do cooperado a partir dos 
investimentos em pesquisa agropecuária, foi definido pelo grupo, iniciar o trabalho em relação aos Investimentos em Pesquisa na perspectiva do Aprendizado e finalizar com o objetivo Rentabilidade do Cooperado na perspectiva financeira. Finalizados os debates e com as definições em torno dos objetivos e dos indicadores, o pesquisador compilou as informações, chegando ao mapa estratégico final. Como pode ser visto na Figura 5 , foram definidos 32 indicadores no total.

Figura 5: MAPA ESTRATÉGICO DA CADEIA DO TRIGO: ESTRUTURA PROPOSTA

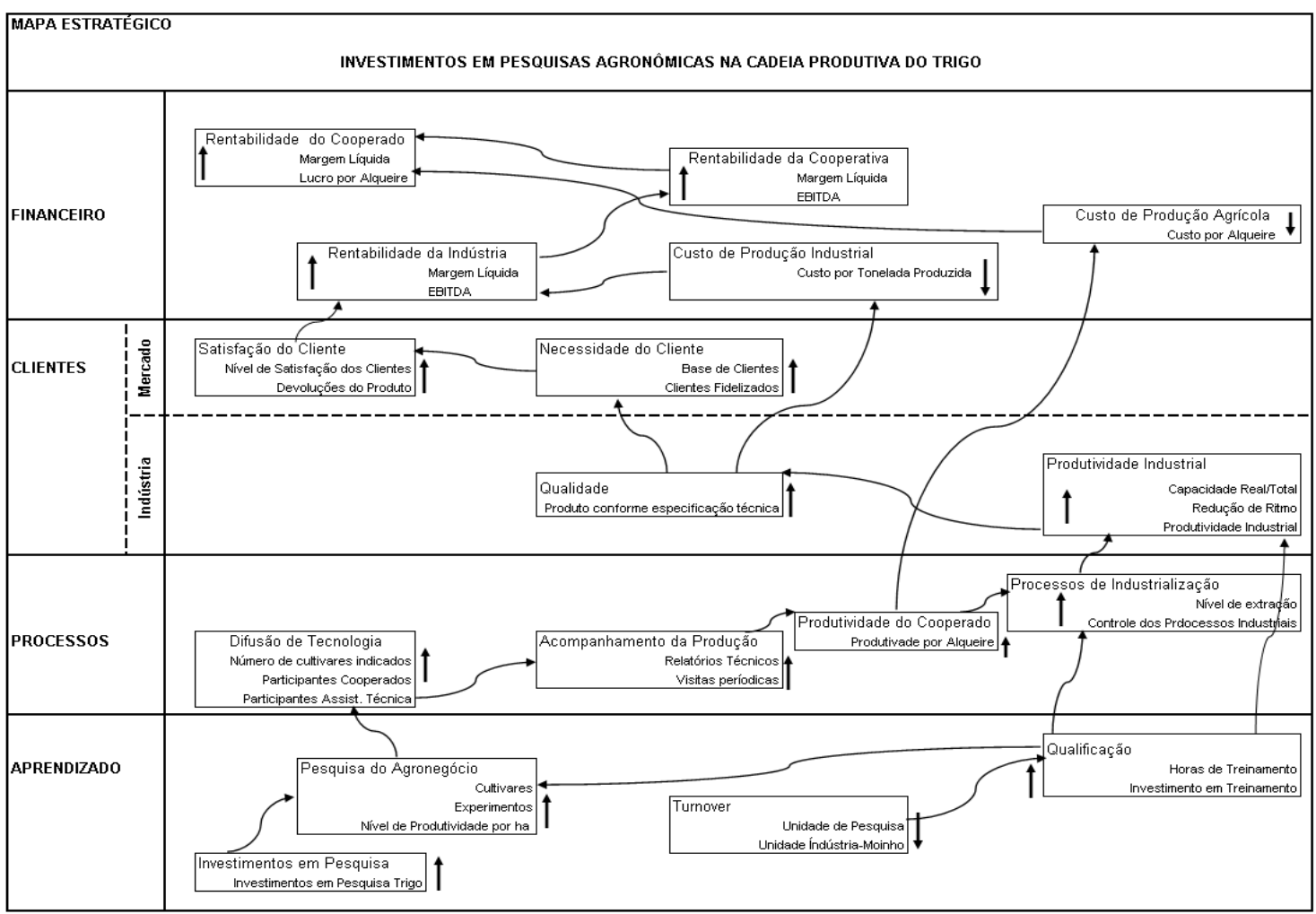

O mapa estratégico do BSC fornece um modelo que mostra como a estratégia liga os ativos intangíveis ao processo que gera valor para a organização. A arquitetura de causa e efeito interconecta os objetivos nas quatro dimensões, tornando claro o relacionamento multidimensional da estratégia (KAPLAN \& NORTON, 1997). A dimensão financeira descreve os resultados tangíveis da estratégia em termo financeiros tradicionais. As medidas da dimensão financeira da cadeia produtiva do trigo definidas foram: rentabilidade do Cooperado, rentabilidade da cooperativa, rentabilidade da indústria, custo de produção industrial e custo da produção agrícola. São indicadores com objetivos de mostrar se a estratégia definida pela organização está no rumo traçado. A dimensão de cliente define a proposição de valor para os clientes, criando um contexto para que os ativos intangíveis criem valor para a organização. As medidas da dimensão clientes são: satisfação do cliente, necessidade do cliente, qualidade e produtividade industrial. A dimensão dos processos internos identifica os processos críticos que influenciam diretamente a estratégia traçada. As medidas na dimensão dos processos internos são: industrialização, produtividade agrícola, controle da produção agrícola e difusão de tecnologia. A dimensão de aprendizado define os ativos intangíveis considerados os principais para a estratégia da organização. Os objetivos dessa dimensão devem ser conectados coerentemente uns com os outros e alinhados aos processos internos do mapa estratégico. As medidas na dimensão de aprendizado são: pesquisa do agronegócio, qualificação, turnover e investimentos em 
pesquisas. Os objetivos nas quatro dimensões são ligados uns com os outros por relações de causa e efeito. O mapa estratégico inicia-se com a hipótese de que os resultados financeiros apenas serão conquistados se os clientes principais estiverem com suas necessidades atendidas e satisfeitos com os serviços recebidos. Já os processos internos desenvolvem e cumprem a proposição de valor para os clientes, e os objetivos da dimensão de aprendizado sustentam a base da estratégia traçada pela organização (FERNANDES, 2001).

\section{CONSTRUÇÃO DO DIAGRAMA DE ENLACE CAUSAL}

O modelo mental apresentado na Figura 6 foi desenvolvido a partir das percepções dos atores envolvidos nesse trabalho. 0 modelo desenvolvido é uma abstração da realidade vivenciada no ambiente de estudo. De acordo com Senge (2004) todos os modelos são simplificações da realidade vivenciada, das observações e dos conhecimentos adquiridos ao longo do tempo no ambiente estudado. Para compreender melhor a estrutura da cadeia do trigo analisada, foi desenvolvido um mapa qualitativo na estrutura dos conceitos da DS.

O Diagrama de Enlace Causal apresenta o modelo mental da cadeia do trigo, como são as inter-relações existentes no contexto estudado, suas causalidades positivas e negativas verificando os atrasos (delays) existentes no sistema como um todo. Segundo Senge (2004), as organizações que trabalham com modelos mentais, inicialmente precisam desenvolver habilidades de aprendizagem para a implementação de inovações organizacionais na prática do dia a dia. 0 modelo metal é desenvolvido a partir da percepção e um evento e dos acontecimentos vivenciados pelos indivíduos, e é considerado como uma interpretação interna da maneira para analisar um novo evento (DE GEUS, 1998). Dessa forma, as organizações conseguem avaliar seus modelos gerenciais, gerando uma visão do todo do negócio, para suporte ao processo decisório.

FIGURA 6: MODELO MENTAL PROPOSTO PARA A CADEIA PRODUTIVA DO TRIGO

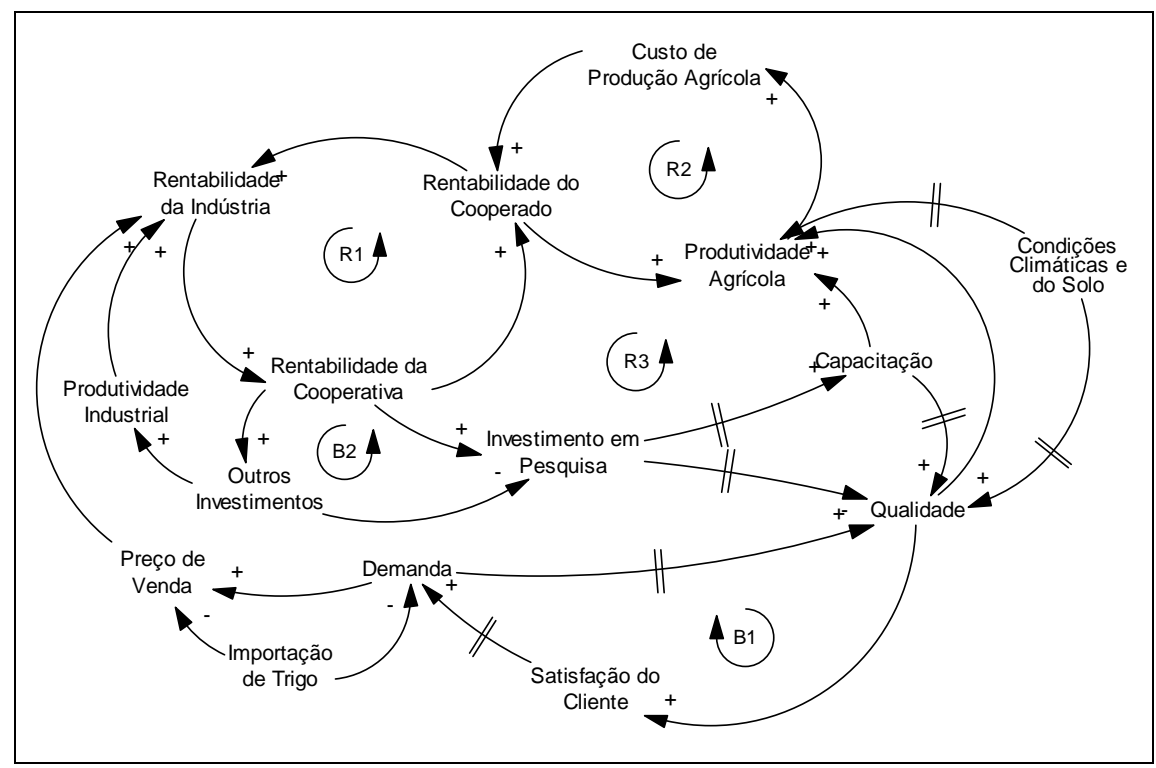

Porém, os modelos mentais não conseguem processar ou interpretar sistemas 
complexos com um grau de variedade e de complexidade, considerando-se não-lineares e os atrasos de tempo entre causa e efeito, com isso, é difícil encontrar soluções intuitivas para determinadas situações (LOURENZANI, 2006). A cadeia do trigo na visão do cooperado pode ser considerada um sistema complexo e também de difícil resolução intuitiva. Assim, na seqüência do trabalho foi desenvolvido um modelo, baseado na estrutura de Fluxos e Estoques, com o objetivo de verificar as variáveis chaves que influenciam no comportamento do sistema e as conexões de causa e efeito circulares existentes.

\section{CONSTRUÇÃO DO MAPA DE FLUXOS E ESTOQUES}

A partir do Diagrama de Enlace Causal foi desenvolvido o diagrama de Fluxos e Estoques apresentado na Figura 7, no qual é possível verificar as principais variáveis levantadas da cadeia do trigo na visão do cooperado. Nessa figura são abordadas as variáveis que ligam as etapas ou elos da cadeia do trigo estudada. No modelo conceitual proposto para a cadeia produtiva do trigo apresentado na estrutura de Estoque e Fluxo é possível verificar como as interligações existentes entre as variáveis chaves, as taxas que controlam essa estrutura e os estoques que gerenciam os elos da cadeia, afetam os resultados financeiros da Cooperativa, da Indústria-Moinho e do Cooperado. Portanto, esse mapa tem como principal objetivo entender a consequências sistêmicas das decisões dos próprios cooperados na assembléia geral para a definição referente à distribuição das sobras operacionais da Cooperativa. Conforme o padrão observado, a decisão tomada na assembléia geral da Cooperativa sobre o repasse dos recursos destinados aos investimentos em pesquisas do agronegócio pode afetar o desempenho de toda a cadeia do trigo e impacta diretamente na rentabilidade do Cooperado. 
FIGURA 7: DIAGRAMA DE ESTOQUE E FLUXO DA CADEIA DO TRIGO

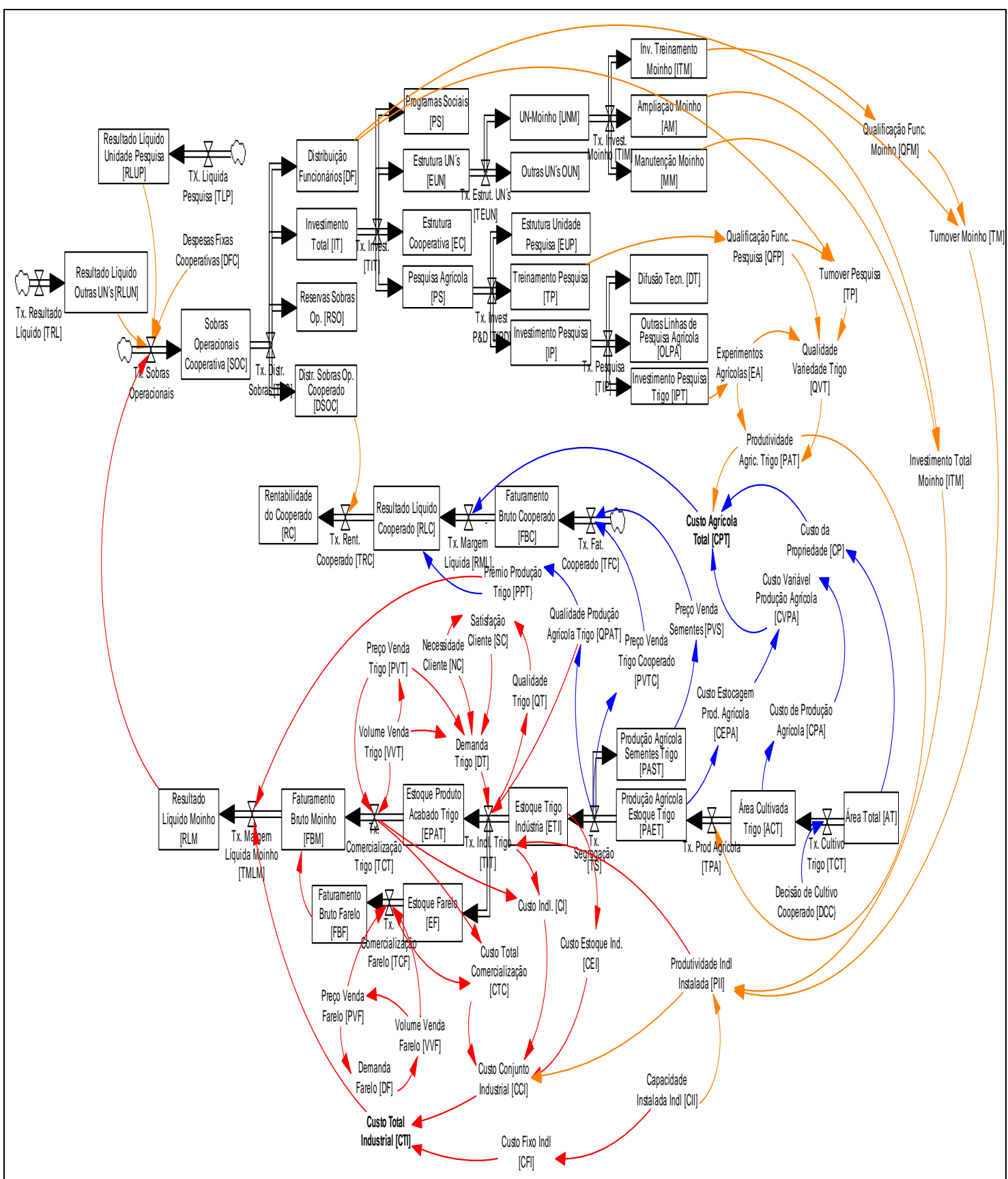

A fim de conectar os objetivos estratégicos do Scorecard da Cooperativa com a dinâmica da estrutura do sistema foi elaborado o modelo da Figura 8. Percebe-se que todos os objetivos e indicadores do BSC são gerados a partir dos resultados das variáveis chaves do sistema conforme a estrutura apresentada a partir dos conceitos da Dinâmica de Sistemas (DS). 
FIGURA 8: INTEGRAÇÃO DA DINÂMICA DE SISTEMAS (DS) AO BALANCED SCORECARD (BSC)

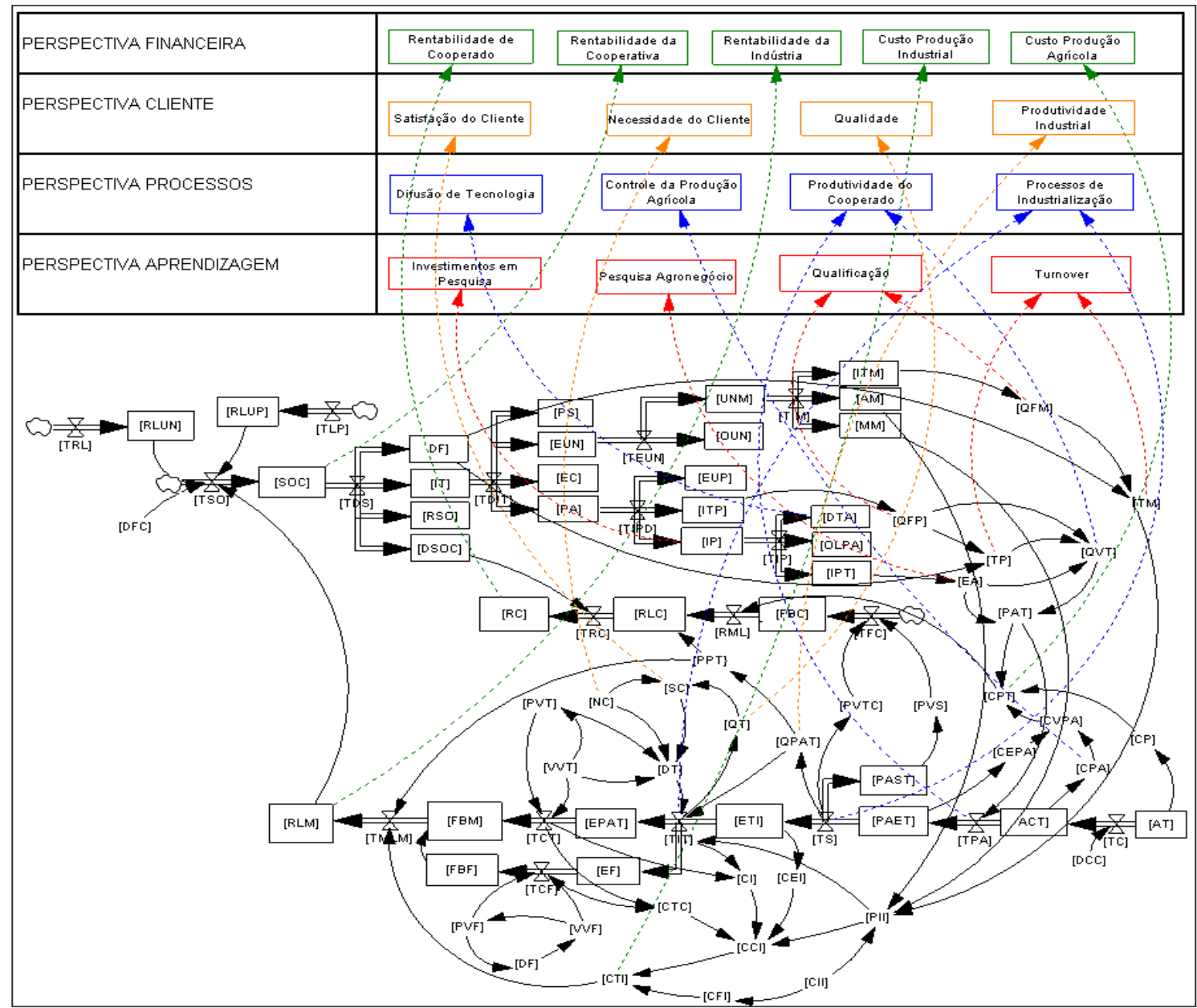

\section{DISCUSSÃO DO MODELO CONCEITUAL PROPOSTO}

A primeira etapa operacional definida para a resposta do objetivo geral deste trabalho foi o levantamento da estrutura da organização em estudo. Esse levantamento norteou a execução das demais etapas, porque proporcionou um melhor entendimento do universo em que a unidade estudada está inserida sendo possível assim entender com mais profundidade as peculiaridades da estrutura da Cooperativa.

O mapeamento da cadeia do trigo na visão cooperado foi fundamental para a compreensão do sistema como um todo, verificando os elos existentes e suas inter-relações ao longo da estrutura. Com os avanços e aprofundamento dos estudos na organização, em conjunto com os atores envolvidos nesse trabalho, foram surgindo novas variáveis, e estabelecidas ligações que auxiliaram a projetar o comportamento do sistema. Após o mapeamento da cadeia do trigo na visão do cooperado, foram levantadas algumas situações de interdependência entre a Unidade de Pesquisa no Agronegócio e a IndústriaMoinho.

Conforme foi se avançando na modelagem da estratégia, percebeu-se que o mapa do BSC é relevante para o controle e monitoramento da estratégia de investimentos em pesquisa do agronegócio. Para cada perspectiva foram desenvolvidos objetivos estratégicos que controlassem e monitorassem os resultados parciais da cadeia do trigo até a 
maximização do objetivo financeiro de rentabilidade do cooperado. Os objetivos do mapa estratégico foram ligados nas quatro perspectivas, com a intenção de analisar o impacto do resultado de um determinado objetivo no desempenho de outro. Essas inter-relações observadas e validadas pelos atores envolvidos no trabalho foram importantes para a identificação dos processos chaves ou críticos do mapa estratégico para o sucesso da cadeia do trigo como um todo.

Para uma melhor representação da realidade, a perspectiva de cliente foi subdividida entre Indústria-Moinho e Mercado. A sub-divisão Indústria-Moinho depende do fornecimento da produção agrícola com qualidade por parte do cooperado para que o processo de industrialização do trigo atenda aos padrões de qualidade e níveis de produtividade esperados. A matéria-prima, o trigo, que o cooperado repassa para a Indústria-Moinho precisa seguir padrões de qualidade definidos internamente e outros definidos pelo próprio cliente, seguindo o propósito de manter um desempenho dentro do esperado nos objetivos de Produtividade e nível de Qualidade. Caso os dois objetivos da perspectiva Cliente Indústria-Moinho não apresentem resultados conforme metas estipuladas, o impacto será negativo nos objetivos Cliente-Mercado. Com o não atendimento das Necessidades do Cliente e o desempenho no objetivo de Satisfação do Cliente ficará aquém das expectativas. Os resultados abaixo do esperado nos objetivos do Cliente-Mercado impactam negativamente no desempenho dos objetivos financeiros do Mapa Estratégico apresentado.

Como o orçamento destinado à pesquisa agropecuária é limitado, os gestores precisam avaliar todos os projetos de pesquisas e decidir quais poderão gerar um melhor resultado para a Cooperativa como um todo. Com o direcionamento dos investimentos em pesquisas agropecuárias, a Cooperativa do caso vem conseguindo gerar avanços nas variedades a serem cultivadas na região onde atuam, melhorando os índices de produtividade agrícola, a redução dos custos de produção agrícola e principalmente reduzindo os impactos ao meio ambiente. Através das modelagens foi possível verificar que os investimentos em pesquisa serão inócuos se não uma houver correspondente difusão de tecnologia, primordial para que os Cooperados consigam índices de produtividade agrícola próximo aos obtidos experimentalmente. A responsabilidade da difusão de tecnologia cabe à Assistência Técnica, que fará o controle e monitoramento desde a decisão da escolha da variedade a ser cultivada em determinada região até o processo de controle e acompanhamento da produção agrícola junto com o Cooperado. Esse processo é importantíssimo para que a Indústria-Moinho receba o trigo do Cooperado com a qualidade necessário para atender as necessidades de sua carteira de clientes.

A Indústria-Moinho ao receber a produção agrícola, tem a tarefa de segregar a produção conforme classificação interna. Após esse processo de segregação e estocagem da produção agrícola, inicia-se a etapa de industrialização do trigo. Essa etapa de industrialização do trigo para atender a necessidade do cliente segue padrões de qualidade rigorosos, para não entregar ao cliente um produto fora das especificações solicitadas inicialmente. Com o desenvolvimento dos objetivos nas perspectivas propostas, foram identificados dois processos críticos no mapa estratégico desenvolvido na cadeia do trigo que poderão influenciar negativamente nos resultados de rentabilidade. 0 primeiro processo crítico envolve as etapas da difusão de tecnologia, controle da produção e da produtividade agrícola. E o segundo processo crítico identificado afeta as etapas de Industrialização do Trigo e a Produtividade Industrial. Caso o resultado do primeiro processo crítico da cadeia do trigo, na visão do cooperado, ficar aquém do esperado, os resultados dos demais objetivos também serão influenciados. Como os objetivos estão inter-relacionados, qualquer desempenho abaixo da meta, direcionará para uma possível redução do índice de rentabilidade do cooperado. 
Com a estrutura do mapa estratégico da cadeia do trigo formalizada, os atores envolvidos nesse processo perceberam a importância de desenvolver um novo modelo de gestão para medir os possíveis impactos dos investimentos em pesquisa do agronegócio na rentabilidade do cooperado. 0 ganho gerencial que o mapa estratégico da cadeia do trigo gerou foi a percepção da existência de relações de causa e efeito não linear entre os objetivos estratégicos da organização e como esses objetivos inter-relacionados levam ao alcance do indicador macro, aumento da Rentabilidade do Cooperado. Com isso, ações tomadas sem a percepção do todo da cadeia do trigo pode gerar resultados positivos em determinados objetivos no curto período de tempo, mas, no futuro, os impactos nos resultados e nos demais objetivos podem não ser atingidos, resultando num comportamento totalmente oposto ao previsto inicialmente.

Com o modelo conceitual de fluxos e estoques, percebe-se mais claramente como as principais variáveis influenciam no comportamento do sistema e como os investimentos realizados pela Cooperativa levam ao ganho de rentabilidade da Indústria-Moinho e do Cooperado. Em relação aos investimentos destinados para a Indústria-Moinho, verifica-se que a partir das inter-relações existentes entre as variáveis chaves do sistema é possível dimensionar o impacto no desempenho na produtividade industrial, causado pelos investimentos na estrutura realizados para manutenção do parque industrial atual e também para a ampliação da capacidade instalada de processamento do trigo. Essas decisões de investimentos em manutenção e ampliação do parque industrial podem levar a uma redução no custo conjunto de produção. Outro fator que influencia diretamente no aumento do índice de produtividade industrial é a qualificação e a motivação dos funcionários dessa Unidade de Negócio (UN) pesquisada.

Outra constatação fruto da modelagem é que o ganho industrial resultado dos investimentos realizados ao longo do tempo tendem a levar a um melhor desempenho operacional, que influencia diretamente o resultado final da UN. No entanto, este melhor resultado no desempenho operacional não ocorre de imediato, existem alguns atrasos (delays) no sistema, até que os índices operacionais alcancem os níveis desejados ou traçados inicialmente.

Já os investimentos realizados pela Cooperativa repassados a Unidade de Pesquisa no Agronegócio são destinados para estrutura dos laboratórios e equipamentos agrícolas adequados ao manejo dos experimentos, treinamento aos funcionários e para as linhas de pesquisas atuais. Tais investimentos influenciam a qualidade dos experimentos ou dos ensaios agrícolas realizados pelos pesquisadores, nesse caso na linha do trigo. Os investimentos em treinamento impactam na variável experimento agrícola que, por sua vez, influencia o comportamento das variáveis qualidade da variedade de trigo e produtividade agrícola do trigo que está inter-relacionada com a variável taxa de custo agrícola e na taxa de produtividade agrícola. A variável qualidade da variedade do trigo também influencia no resultado da produção agrícola do trigo.

Além dos investimentos em treinamento dos funcionários da Unidade de Pesquisa que elevam o nível de qualificação, também influenciam no nível de motivação reduzindo o índice de turnover pesquisa. 0 programa de distribuição das sobras operacionais para os funcionários também impacta no comportamento da variável turnover pesquisa. Caso o pesquisador agrícola do trigo se desligue da equipe, a unidade de Pesquisa levará em média dois anos para formar um novo pesquisador com o mesmo nível de conhecimento que o pesquisador anterior possuía sobre a cultura do trigo. Conforme informação repassada pelo responsável da Unidade de Pesquisa existe uma preocupação entre os gestores da Unidade de Pesquisa em reter seus talentos nas diversas linhas de pesquisa, com objetivo de evitar resultados aquém das expectativas com os experimentos agrícolas. Caso os resultados com os experimentos agrícolas fiquem abaixo do esperado, ele impacta diretamente na cadeia do trigo, reduzindo a rentabilidade do cooperado e da Indústria- 
Moinho e, por fim, da Cooperativa.

\section{CONSIDERAÇÕES FINAIS}

O presente trabalho tinha como propósito propor um modelo conceitual para explicitar as variáveis e relacionamentos, auxiliando os gestores no processo decisório em relação aos investimentos em pesquisas agropecuárias e na avaliação dos resultados gerados a partir desses investimentos na rentabilidade do Cooperado e da Cooperativa. As pesquisas realizadas com objetivo de compreender a interdependência de diversas variáveis e fatores na organização do caso levaram a construção dos modelos do Balanced Scorecard e da Dinâmica de Sistemas para a cadeia produtiva do trigo.

Foi possível observar que tais modelos geraram uma mudança de atitude dos atores envolvidos no desenvolvimento do trabalho, em relação à visão da cadeia produtiva do trigo, à medida que foram percebidas as inter-relações existentes entre os elos da cadeia estudada e sua importância para o crescimento da Cooperativa. Com o mapa estratégico do BSC, os atores perceberam a importância de mensurar os impactos dos investimentos em pesquisas agropecuárias nos resultados financeiros de toda a cadeia estudada. Com o desenvolvimento do modelo de Dinâmica de Sistemas, foi possível explicitar as ligações de causa e efeito não-lineares e os delays identificados na estrutura da Cooperativa.

O modelo proposto do BSC para a cadeia do trigo também contribuiu para que os atores envolvidos no trabalho desenvolvessem uma nova visão estratégica sobre a cadeia produtiva do trigo. Como, a partir do mapa estratégico eles poderão mensurar os resultados dos investimentos em pesquisas agrícolas na rentabilidade do cooperado, alguns processos críticos identificados deverão ser controlados para gerar uma estratégia sustentável nos mercados onde a cooperativa atua.

A partir do mapa de Fluxos e Estoques da cadeia do trigo na visão do cooperado, foram apresentadas a estrutura e as ligações existentes entre as variáveis chaves, as taxas que governam os fluxos, os estoques e os delays do sistema estudado, criando as condições para a simulação de estratégias. Com a parametrização do diagrama de estoque e fluxo será possível criar uma plataforma que possa suportar a simulação dos investimentos em pesquisa do agronegócio, permitindo assim que os gestores avaliem o impacto destes investimentos na rentabilidade futura do cooperado.

Finalmente, o grupo de atores envolvido na pesquisa da cooperativa emitiu a seguinte opinião: "para a Cooperativa e a Unidade de Pesquisa é importante o conhecimento de novas ferramentas de gestão da produção. 0 estudo em questão foi necessário para que se tivesse a visão detalhada de todos os elos da cadeia produtiva do trigo, bem como as implicações entre eles e a importância de todos para a Cooperativa. Temos a convicção de que o uso desta ferramenta será vital para a concretização da Missão e Visão da Cooperativa".

\section{REFERÊNCIAS}

ABAG - Associação Brasileira de Agribusiness. Superávit e Exportações do Agronegócio batem recorde nos últimos 12 meses. Disponível em: <http://abag.com.br>. Acesso em 23 maio 2005. 
Dados de Mercado. Disponível em: http://abag.com.br. Acesso em 10 jun. 2006.

AKKERMANS, H.; OORSCHOT, K. Van. Developing a Balanced Scorecard with System Dynamics. Journal of the Operational Research Society, may, 2002.

BIALOSKORSKI NETO, S, N. Agronegócio cooperativo. In: BATALHA, M. O. Gestão Agroindustrial. São Paulo, p. 628-655, 2001.

CASTRO, A. M. G. de. Análise da Competitividade de Cadeias Produtivas. Manus, 2000. Workshop Cadeias Produtivas e Extensão Rural na Amazônia, promovido pela Suframa. Disponível em: <http://www.suframa.gov.br/download/publicacoes/accp.pdf>. Acesso em 19 out. 2005.

DE GEUS, A., Foreword: Modeling to Predict or to Learn? In: MORECROFT, John D. W. \& STERMAN, John D. Modeling for Learning Organizations. Portland, Productivity Press, p. xiii-xvi,1994.

FERNANDES, A. da C. Dinâmica de Sistemas e Business Dynamics: tratando a complexidade no ambiente de Negócios. Encontro Nacional de Engenharia de Produção - ENEGEP, Anais do XXI Encontro Nacional de Engenharia de Produção, Bahia, outubro de 2001.

.Scorecard Dinâmico: em Direção à Integração da Dinâmica de Sistemas com o Balanced Scorecard. 320f. Tese de Doutorado em Engenharia de Produção - Escola de Engenharia, COPPE - UFRJ, Rio de Janeiro, 2003.

KAPLAN, R. S.; NORTON, D. P. Mapas Estratégicos: convertendo ativos intangíveis em resultados tangíveis. Rio de Janeiro: Campus, 2004.

.A Estratégia em Ação: balanced scorecard. Rio de Janeiro: Campus, 1997.

LINARD, K., BASSET, M., DVORSKY, L., YOON, J., A dynamic balanced scorecard template for public sector agencies. International Conference of the System Dynamics Society, Proceedings of the $18^{\text {th }}$ International Conference of the System Dynamics Society, Bergen, August, 2000.

LOURENZANI, W. L. Modelo Dinâmico para a gestão integrada de Empreendimentos Rurais. Congresso Internacional de Dinâmica de Negócios, Anais do $1^{\circ}$ Congresso Internacional de Dinâmica de Negócios, Brasília, setembro, 2006.

OCEPAR - Organização das Cooperativas do Estado do Paraná. Sistema OCEPAR. Disponível em <www.ocepar.org.br> . Acesso em 21 out. 2005.

Participação das cooperativas na produção agropecuária do Paraná em 2005. Disponível em <www.ocepar.org.br> Acesso em 7 fev. 2007.

RICHMOND, B. A New Language for Leveraging Scorecard-Driven Learning. Harvard Business School Publishing. Balanced Scorecard Report, 2001.

Using the Balanced Scorecard to Leverage Penetration of Systems Thinking. Hanover, High Performance Systems, 1999.

ROSSI, R. M.; NEVES, M. F. Estratégias para o trigo no Brasil. São Paulo: Atlas, 2004.

SENGE, P. M. A quinta disciplina: arte e prática da organização que aprende. 16. ed. São Paulo: Best Seller, 2004.

TODD, D., PALMER, E., Development and Design a Dynamic Scorecard in Local Government, 2000. Disponível em: «www.synergia.co.nz». Acesso em 29 abr. 2007.

ZYLBERSZTAJN, D. Quatro Estratégias Fundamentais para Cooperativas Agrícolas. Série Working Papers - FEA - USP, São Paulo. Disponível em <www.ead.fea.usp.br/wpapers>, 2003. 


\section{ENDEREÇO DOS AUTORES}

Pontifícia Universidade Católica do Paraná

Escola de Negócios

R. Imaculada Conceição, 1155

Prado Velho

Curitiba, PR - Brasil

80215-901

Universidade Federal do Rio de Janeiro

Escola de Engenharia

Departamento de Engenharia Industrial

Centro de Tecnologia

Fv. Brigadeiro Trompowski, s/ no, sala F117

Cidade Universitária

Rio de Janeiro, RJ - Brasil

21949-900

Universidade Federal do Ceará

Departamento de Contabilidade

R. da Universidade, 2431 - Benfica

Caixa-Postal: 12131

Fortaleza, CE - Brasil

60020-180 